\title{
Derived Synovial Fluid Cell
}

National Cancer Institute

\section{Source}

National Cancer Institute. Derived Synovial Fluid Cell. NCI Thesaurus. Code C138971.

Cells derived from the fluid found in the cavities of synovial joints. 